Manuscript prepared for Biogeosciences

with version 5.0 of the $\mathrm{L}_{\mathrm{T}} \mathrm{T}$ class copernicus.cls.

Date: 23 July 2018

\title{
The Habitable Zone of Inhabited Planets
}

\author{
Jorge I. Zuluaga Callejas ${ }^{1}$, Juan F. Salazar ${ }^{2}$, Pablo Cuartas-Restrepo ${ }^{1}$, and German Poveda ${ }^{3}$ \\ ${ }^{1}$ FACom - Instituto de Física - FCEN, Universidad de Antioquia, Calle 70 No. 52-21, Medellín, Colombia \\ ${ }^{2}$ Escuela Ambiental, grupo GIGA, Facultad de Ingeniería Universidad de Antioquia, Calle 70 No. 52-21, Medellín, Colombia \\ ${ }^{3}$ Escuela de Geociencias y Medio Ambiente, Universidad Nacional de Colombia, Medellín, Colombia
}

Correspondence to: Jorge I. Zuluaga

(jzuluaga@ fisica.udea.edu.co)

\begin{abstract}
In this paper we discuss and illustrate the hypothesis that life substantially alters the state of a planetary environment and therefore, modifies the limits of the $\mathrm{HZ}$ as estimated for an uninhabited planet. This hypothesis lead to the introduction of the Habitable Zone for Inhabited planets (hereafter InHZ), defined here as the region where the complex interaction between life and its abiotic environment is able to produce plausible equilibrium states with the necessary physical conditions for the existence and persistence of life itself. We support our hypothesis of an InHZ with three theoretical arguments, multiple evidences coming from observations of the Earth system, several conceptual experiments and illustrative numerical simulations. Conceptually the diference between the InHZ and the Abiotic HZ (AHZ) depends on unique and robust properties of life as an emergent physical phenomenon and not necesarily on the particular life forms bearing in the planet. Our aim here is to provide conceptual basis for the development of InHZ models incorporating consistently life-environment interactions. Although previous authors have explored the effects of life on habitability there is a gap in research developing the reasons why life should be systematically included at determining the HZ limits. We do not provide here definitive limits to the InHZ but we show through simple numerical models (as a parable of an inhabited planet) how the limits of the AHZ could be modified by including plausible interactions between biota and its environment. These examples aim also at posing the question that if limits of the $\mathrm{HZ}$ could be modified by the presence of life in those simple dynamical systems how will those limits change if life is included in established models of the AHZ.
\end{abstract}

Keywords. Habitable Zone; Habitability; Planetary Habitability and Biosignatures; Planetary Environments

\section{Introduction}

"It can scarcely be denied that the supreme goal of all theory is to make the irreducible basic elements as simple and as few as possible without having to surrender the adequate representation of a single' datum of experience"

Albert Einstein, 1934

The search for life outside the Solar System has nowadays reaching the level where almost two thousand of extrasolar planet $\$ 1$ and even more exoplanet candidates (Batalha et al. 2013) have been discovered. Assesing the potential of these worlds to host surface liquid water (generally assumed as the most important physical prerequisite for life) is important and for that purpose it has been introduced the concept of a "habitable zone" (HZ) (Dole 1964; Hart 1979; Kasting et al. 1993). Traditionally, the definition of habitability has been mainly related to planetary insolation, i.e. the equilibrium between the amount of radiation a planet receives from its parent star and the energy the planet radiates to space from its surface and atmosphere. Planetary insolation is supposed to determine the capacity of a planetary environment to harbor surface liquid water and hence an evolving and, probably more importantly, detectable biosphere. A purely isolation condition leads straightforwardly to the concept of a Radiative Habitable Zone (RHZ) (Kasting et al. 1993), defined as the spherical shell around a star where insolation, provided the planet have a dense enough atmosphere, is compatible with surface liquid water and probably with life as we know it.

Nowadays the definition of the HZ has trascended the pragmatical goal of simply selecting which candidates in a exoplanetary survey could be further studied. Habitability has morphed in a complex and probably more fundamental

\footnotetext{
${ }^{1}$ For updates please refers to: http://exoplanet.eu/
} 
subject involving the unique properties of life and its interaction with a dynamical planetary environment.

The definition the HZ, either obeying insolation or other complex physical factors, assumes habitability as a necessary, although not sufficient abiotic condition for life. However, and as the observation of the Earth System (ES) suggests, habitability is not only an abiotic prerequisite but an emergent property of a very complex system involving the interaction among astrophysical, geophysical and not less important biological factors (Sagan and Mullen 1972; Lovelock and Margulis 1974; Margulis and Lovelock 1974; Walker et al. 1981; Franck et al. 2000bla, 2001; Franck 2001; Lovelock 2009; Rosing et al. 2010).

The effect that life has at determining the equilibrium state of a habitable planet has been much less studied when compared with the effects of many other abiotic factors. This is especially true when dealing with the estimation the limits of the $\mathrm{HZ}$ in extrasolar planetary systems.

The key role of life in the environment has been widely discussed in the literature of the ES (Caldeira et al. 1992; Lenton 2002; Kleidon 2009, 2010a, 2012). In the astronomical community, several works, including the seminal paper by Kasting et al. (1993), have also posed and discussed the importance of life at affecting planetary habitability (for a recent review Kasting (2010) and references therein). However, and as far as we know it, the most consistent efforts attempting to include the effect of biota on the long-term evolution of Earth-analogues' habitability were those made more than a decade ago by S. Franck and collaborators (Franck et al. 1999, 2000b a, 2001; Franck 2001). More recently Dyke et al. (2011); Höning et al. (2013) has also explored the effects of life in the evolution of several geophysical factors affecting planetary habitability. Despite these important efforts, a conceptual basis for the general definition of a Habitable Zone for actually Inhabited Planets, is still lacking. This is clearly evidenced in the absence of biotic factors in most, if not all recent habitability models.

In the case of the Abiotic HZ (AHZ), the lack of a statistically significant number of observations able to confirm the HZ limits predictions, required in the past the development of a solid conceptual basis to support scientifically the theoretical models on which predictions rely. Analogously the discussion of the role of life on habitability requires the development of a general conceptual basis before implementing specific models attempting to redefine the HZ limits.

In order to develop that conceptual basis, we define and discuss here the concept of a Habitable Zone of Inhabited Planets (hereafter InHZ). We support our definition in theoretical arguments based on the understanding of the biotaenvironment interaction as observed in the ES. Since our Planet is the only habitable planet we know so far, its properties are the only point of reference we have for this construction. This is analogous to the way as the Solar System rocky planets (Venus and Mars) are used in the definition of the RHZ limits. Furthermore, we show, through a conceptual experiments and numerical simulations, how the InHZ limits would change with respect to the AHZ in hypothetical inhabited planetary environments.

It is important to stress that our approach does not intend to give (yet) numerical predictions about the extension of the InHZ in actual planetary systems. Our aim here is to provide a general conceptual basis for the development of models able to estimate these limits. Moreover, by using numerical simulations of idealized inhabited planets we just aim at posing the question that if limits of the HZ could be modified by the presence of life on those simple dynamical systems how will those limits change if life were included also in more sophisticated models of the AHZ.

This paper is organized as follows: in Section 2 we define the InHZ and discuss it in the context of the well known AHZ concept. Section 3 is devoted to develop the theoretical arguments that support the introduction of the InHZ. In Section 4 we present the results of conceptual and numerical experiments of the biota-environment interaction that illustrate quantitativeley the InHZ definition. Section 5 is devoted to discuss the limitations, open questions and consequences of pursuing the more general InHZ as opossed to a the AHZ. Finally in Section 6 we summarize our proposal and draw some conclusions and future prospects of this work.

\section{Defining the Habitable Zone of Inhabited Planets}

We define the Habitable Zone of Inhabited Planets (InHZ) as the region (in space and time) where the complex interaction between life and its abiotic planetary environment is able to produce plausible equilibrium states with the necessary physical conditions for the existence and persistence of life itself. This definition does not intend to replace the definition of the AHZ but to extend it.

The reason why life is so important at determining the habitability of a planet lies on its capacity to substantially alter its abiotic environment. For instance, on Earth, biogenic mass fluxes strongly alter the atmospheric structure and composition at different spatial and temporal scales (e.g. Beerling 2005; Pöschl et al. 2010). In the simulations published by S. Franck and collaborators, almost 14 years ago, the inclusion of some biotic feedbacks in the carbonate-sillicate cycle modified substantially the life span of the biosphere in Earth analogues. These examples clearly suggest that life allows the emergence of planetary equilibrium states that would not be predictable if neglecting its effects. In other words, a habitable planet without life and the same planet actually inhabited by a widespread biota are very different, especially in their potential to give rise to plausible habitable equilibrium states.

An inhabited planet is a complex system comprising biotic and abiotic components. Taking away life and its powerful feedbacks is as unnatural as removing liquid water or any other major component of the system. Removing key com- 
ponents of a complex system not only perturbates the properties of the system but it could potentially drive the system to qualitatively distinct equilibrium states.

It is worth noticing that our definition of an InHZ does not exclude the requirement of other abiotic prerequisites. To allow the emergence of complex interactions between life and its abiotic environment, a planet could also require a dense enough atmosphere, complex geophysical processes (e.g. plate tectonics, volcanism) or a protective magnetic field (see e.g. Zuluaga et al. 2013). Even in this case, it has been recently recognized that life on Earth not only has altered the evolution of the atmosphere and oceans. Life could also affect interior geological processes and other global planetary factors (Dyke et al. 2011; Höning et al. 2013). Together, all these evidences point out to identify life not only as an important component of an inhabited planetary environment but as a major geological force at all levels. This significant fact was already anticipated, at least in the case of the ES, by Vladimir Vernadsky circa 1920.

Although at first sight the origin of life could be a problem for the definition of an InHZ, this sort of "egg-and-chicken paradox" is almost inevitable when dealing with complex systems. Here, however, it is interesting to notice that at defining the InHZ we would not require to explain the appearance of the first forms of life in the same way as the definition of the AHZ would not require to explain, for instance, the appearance of the first drop of water. We just need to recall that the definition of the RHZ requires liquid water as a prerrequisite for maintaining the Carbon-Sillicate cycle via weathering processes (Kasting et al. 1993). Therefore, in the same way as explaining the origin of liquid water is not mandatory to define the RHZ, explaining the origin of life is not necesarily required to define the InHZ.

\section{Theoretical arguments}

"We are only now beginning to acquire reliable material for welding together the sum total of all that is known into a whole [...] Some of us should venture to embark on a synthesis of facts and theories, albeit with second-hand and incomplete knowledge of some of them - and at the risk of making fools of ourselves" Erwin Schrodinger in "What is Life?” (1992)

There are three key theoretical arguments supporting the idea that habitability should not be assessed without including the influence of life:

(1) Biota-environment feedbacks are likely to substantially alter the equilibrium states of any inhabited planet.

(2) The equilibrium state of a complex system cannot be predicted while neglecting one of its major components (in this case life).
(3) Living phenomena have unique properties, hardly mimicked by abiotic mechanisms and able to explain the maintainance of physically unstable states of inhabited planets.

In the following paragraphs we develop in detail each argument and present the observational and theoretical evidences supporting them.

\subsection{Argument 1: the power of biota-environment feed- backs}

Life alters its environment and the environment constrains life. This well-known two-way relationship implies the existence of biota-environment feedbacks which can produce global scale effects as life forms grow and reproduce (Lenton and Wilkinson 2003; Foley et al. 2003). These global scale feedbacks will be an universal feature of planets inhabited by a widespread biota.

Biota-environment feedbacks can strongly alter the physical conditions that are regularly taking into account when defining abiotically the HZ. Thus, for instance the water and carbon content of the atmosphere or the presence of clouds in the Earth, would not be the same if our planet were uninhabited (Lenton 1998; Lovelock 1995). We argue here that in any inhabited planet the power of such biota-environment feedbacks is too large to be neglected when definining the $\mathrm{HZ}$

Life is based on biochemical reactions that continuously convert inorganic substances stored in the environment into organic ones and back. Therefore, large biochemical fluxes of synthesis and decomposition of organic substances are expected. In the Earth the power of these fluxes is such large that if they were not tightly compensated, the environment could change dramatically in time-scales of several tens of years (Gorshkov et al. 2004). Those changes could bring the environment to a state incompatible with the existence of life itself (Makarieva and Gorshkov, personal communication 2013)

One of the most noticeable biota-environment feedbacks regarding habitability may be those related to clouds. Water or carbon dioxide clouds are key at determining the extension of the HZ (see e.g. Mischna et al. 2000, Kitzmann et al. 2010). On Earth, water clouds are a key component of the climate system, and its influence on the equilibrium state of the environment is presumably large (see, e.g. Ramanathan et al. 1989). Land vegetation and phytoplankton play an important role at controlling the amount of cloud condensation nuclei $(\mathrm{CCN})$ in the atmosphere (Meskhidze and Nenes 2006; Pöschl et al.2010), thereby affecting the formation of clouds. The presence of native vegetation may enhance the formation of clouds especially over certain areas of the planet (Lyons 2002). Even airborne microorganims living in the middleupper troposphere can work as biotic cloud condensation nuclei (DeLeon-Rodriguez et al. 2013). In large scale nat- 
ural forests such as the Amazon, physical connections between clouds, rainfall and vegetation have been also identified (Andreae et al. 2004; Bonan 2008).

Biota-environment interactions on Earth are not restricted to the effects on clouds. Terrestrial biota also plays an important role both in the hydrologic cycle (Huties et al. 1998) and in the global carbon cycle (Schimel 1995). It has been recently shown, for instance, that terrestrial water fluxes are dominated by biological processes (transpiration) rather than physical ones (evaporation) (Jasechko et al. 2013). Moreover, a non negligible number of feedbacks through which forests exerts strong effects on climate regulation have been also identified (Bonan 2008). For instance, it has even been proposed that forest vegetation can interact with its surrounding environment in ways that enhance conditions favorable for its own existence (Runyan et al. 2012). Natural forests may be responsible for a biotic pump of atmospheric moisture driving the hydrologic cycle on land (Makarieva et al. 2007, 2010; Poveda et al. 2014).

Concerning the carbon-cycle, plant evolution on Earth, for example, has strongly influenced the amount of $\mathrm{CO}_{2}$ in the atmosphere at geological timescales (Beerling 2005). Other biological processes such as the ecological success of calcareous plankton have driven important changes in the global carbonate cycle (Ridgwell and Zeebe 2005). These changes have had important implications on atmospheric and ocean chemistry, and hence on the regulation and evolution of the ES at geological timescales (Ridgwell and Zeebe 2005). These evidences have lead to recognize terrestrial biota as a key regulator of the atmospheric chemistry and global Earth climate (Arneth et al. 2010).

In summary biologically driven processes can significatively alter the global biogeochemical and biogeophysical cycles and therefore, the equilibrium habitable state of our planet would not be the same without the effects of biota. Moreover, these effects exist as a part of the complex Earth system, irrespectively of accepting that life plays a determinant role at regulating the environment.

\subsection{Argument 2: Equilibrium States of Inhabited Hab- itable Planets}

The Earth functions as a whole complex system having physical, chemical and biological coupled components. It is not possible to understand the functioning of the ES without considering it as whole (Houghton et al. 2001, p.784; Rial et al. 2004). Interestingly Schellnhuber (1999) refers to modern scientific advances striving to understand the ES as a whole and the development of new concepts on this basis, as a "second Copernican revolution". Turning from an AHZ to an InHZ brings this "revolution" to the search for habitable planets.

Although the knowledge of whether and how life provides an "establishing" influence on the ES remains elusive, there is no doubt that biota plays a crucial role in the complex be- haviour of the system (Steffen 2004, p.69-70). Consequently the plausible equilibrium states of a planetary environment cannot be predicted, especially if inhabited by a widespread biota, without taking into account the role of life. If our goal is to search for life in the universe, the determination of the limits of the HZ, which is essentially based on studying the plausible equilibrium states of the system, must consider the role of life.

We illustrate the key differences between the AHZ and the InHZ in Figure 1 We show there the equilibrium states of uninhabited and inhabited planets. The balls represents the states of planetary environments, while the valleys or potential wells depicted in the left column panels, represent stable atractors either in the case of uninhabited (U) or inhabited (I) planets. An uninhabited planet can be inside the HZ (i.e. the AHZ) if exist at least one plausible equilibrium state where the surface temperature (and other environmental variables) is within the range of values where liquid water can exist (shaded strip). This planet can be also habitable if inhabited, although its equilibrium state can be different (row 3). Inhabited equilibrium states could be characterized by biologically induced oscillations (a limit cycle) rather than states characterized by almost constant values of the environmental variables (fixed points) (in Section 4.1 we provide a specific example of this condition). Rows 1 and 5 illustrate those situations in which abiotic conditions are prohibitively extreme for life, either because the planet is too cold (row 1) or too hot (row 5). The important point here is that there may exist intermediate conditions between cases 1,3 , and 5 , which would be "invisible" without involving biotic activity in the dynamic of the environment. A coupled biota-environment system may be able to find a habitable equilibrium state in a planet which, if uninhabited, would be too cold (row 2) or too hot (row 4) to be habitable. In this sense rows 2 and 4 would represent planets within the InHZ. It is important to stress that these planets would be otherwise considered uninhabitable if the role of life were ignored. Since solar forcing increases from row 1 to row 5 , these results can be interpreted as an stretching of the HZ towards the InHZ. This strectching occurrs either in time (going from row 1 to 5 solar luminosity increases) or in space (going from row 5 to 1 the distance to star increases).

In the examples depicted in rows 2 and 4 of Figure 1 the presence of life in otherwise uninhabitable planets allows the emergence of habitable conditions. But there should also be another possibility. Organisms producing a de-stabilizing effect on the environment (disruptive organisms) could also evolve and induce catastrophic events able to make permanently uninhabitable a planet. They could for instance change the composition of the atmosphere inducing a runaway greenhouse effect or bringing out radioactive elements from planetary interior sterilizing permanently the surface. However, none of these scenarios would be different than other catastrophic abiotic events (an asteroid impact or a nearby supernova). 


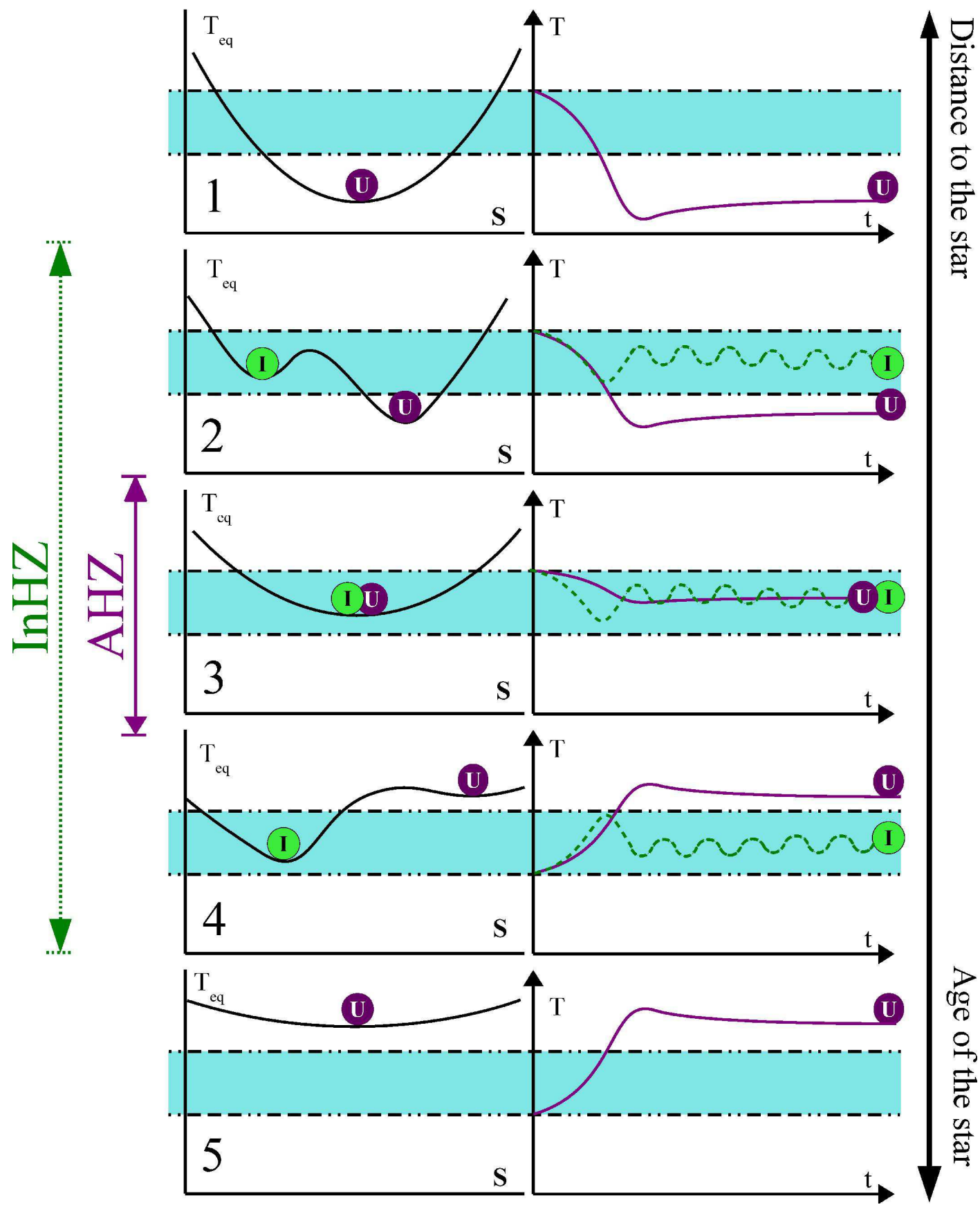

Fig. 1. Conceptual representation of the environmental equilibrium states in uninhabited and inhabited planets. The potential wells represent stable atractors, and the ball the state of the system for an inhabited (I) or uninhabited (U) planet. Right column shows the time evolution of global surface temperature until reaching equilibrium. Equilibrium states can be fixed points, or limit cycles characterized by temperature oscillations. The range of temperature values within wich liquid water can exist is indicated by horizontal dashed lines. Stellar flux increases when moving from row 1 to 5 either in response to the evolution of the stellar luminosity or because we are closer to the star. 
It should be then important to stress that the InHZ, as well as the AHZ, are not defined depending on random events, either physical or biological, but on the existence of plausible habitable equilibrium states in the system under regular (non-transient) conditions. In order to further clarify the independence of habitability on random events we propose the following general definition:

\section{A planet is within the $H Z$ (either the AHZ or the InHZ) if under certain set of regular (non- transient) internal and external conditions, there exists at least one equilibrium state compatible with the existence and persistence of life.}

Accordingly, if those conditions making plausible the existence of habitable equilibrium states involve life, then the $\mathrm{HZ}$ would be an InHZ.

\subsection{Argument 3: The unique properties of life}

The role of life in the determination of the equilibrium state of the ES has been widely discussed and extensively developed by two complementary theories: Gaia (Margulis and Lovelock 1974; Lovelock 1979; Lenton 2002) and Biotic Regulation of the Environment (hereafter BR) (Gorshkov 1995; Gorshkov et al. 2000, 2004)2. Independently and from a purely physicochemical perspective the connection between life and the regulation of the Earth environment has also been explored in the studies of A. Kleidon and collaborators on the non-equilibrium thermodynamics of the ES(Kleidon 2010a b, 2012).

These three independent theoretical frameworks agree that the presence of life on Earth plays a major role at determining the Earth's physically unstable equilibrium state(Margulis and Lovelock 1974; Lovelock 1979; Gorshkov 1995; Gorshkov et al. 2000; Lenton 2002; Gorshkov et al. 2004; Kleidon 2012). Moreover and according to, for instance, BR, life makes the resulting unstable state resilient and biotically stable (A. Makarieva and V. Gorshkov, personal communication, 2013)

An interesting implication of the influence of life on the equilibrium state of the ES is that the lifespan of Earth's biosphere can be extended (Lenton and von Bloh 2001). This result is in agreement with the independent simulations performed by S. Franck and collaborators (Franck et al. 2000a) [e.g.] and with the argument presented in Section 3.2

Together, all these theories and models support the notion that the influence of life cannot be excluded when assesing

\footnotetext{
${ }^{2}$ It is interesting to recall that the original motivation of Gaia theory was precisely the search for extraterrestrial life (Hitchcock and Lovelock 1967; Lovelock 1979). Therefore, the idea that life is somehow involved in the determination of the habitability of a planet has been implicit in the literature since the appearance of the aforementioned works and could even be traced back to the introduction of the biosphere concept by V.I. Vernadsky in 1926.
}

the habitability of inhabited planets. This is because the presence of life confers to the complex system (the inhabited planet) properties that (1) exerts a non nengligible influence on the system's equilibrium and (2) are hardly replaced or mimicked by even very complex abiotic factors.

Lenton (1998) developed several fundamental arguments about why biota can be distinguished of other abiotic factors. In Lenton's own words “in contrast to a dead world, the introduction of organisms brings an inherent tendency to stabilize conditions that are inhabitable by life". This assertion alone agrees with the intrinsic difference between an InHZ and an $\mathrm{AHZ}$ as argued here. According to Lenton, three intrinsic properties of life drive an inhabited planetary environment towards a self-regulated (stationary) habitable state (Lenton 1998):

(1) Organisms alter the environment by taking and excreting energy and waste products. At doing so, life can produce novel biogeophysical and biogeochemical feedbacks (e.g. feedback on growth and feedback on selection, see below) competing with and possibly dominating over the otherwise existing abiotic physicochemical feedbacks.

(2) Organisms grow and multiply, potentially exponentially, leading to global positive feedback on the environment (more individuals means also a larger capacity to grow). Growth tends to amplify any already existing biological feedback.

(3) For each environmental variable there is a level or a range of values whereby a giving organism grows at a maximum rate. This property gives rise to the existence of positive and negative biota-environment feedbacks around the optimum values of the environmental variables. With enough biological amplification, the interplay of those positive and negative feedbacks tends to stabilize the whole system.

Life is also unique because it can produce two kind of feedbacks not present on abiotic systems, namely feedback on growth and feedback on selection. These novel feedbacks can have a large effect on the regulatory capacity of the system Lenton (1998, 2004). The feedback on growth occurs when an organism induces changes in the environment that affects in the same way the growth of every competing organism, so no selection force is induced. The feedback on selection occurs when the changes an organism introduces in the environment affect distinctly each specie creating a selection force: organisms which are affected in the sense that their growth is reduced under the modified conditions tend to disappear. On the other hand, the species being favored by the change are selected and stay alive. Feedback on growth and feedback on selection are hardly found in other abiotic complex systems.

Independently, V.G. Gorshkov and A. Makarieva Gorshkov and Makarieva 2001, 2002; Gorshkov et al. 
2004; Makarieva et al. 2006) identify other two unique properties of living systems confering life unparallel capacities with respect to the abiotic world (Gorshkov et al. 2004):

(4) "Living matter features a level of orderliness incomparably higher than than the surrounding environment" (Gorshkov et al. 2004).

(5) "Life supports its orderliness in a way unprecedented in the inanimate world: by competitive interaction" (Gorshkov et al.2004)

According Gorshkov and Makarieva the large effect of life on the regulation of the environment (biotic regulation), i.e. the maintanance of the unstable habitable equilibrium state, results from the correlated functioning of organisms that form local ecological communities (Gorshkov et al. 2004). These correlations depend on information stored in the genomes of biological species. In non-living open physical systems a similar link between their regulatory capacity and information can be established. In this case information is associated to the degree of orderliness in the system (number of available degrees of freedom). Gorshkov and Makarieva (2001) have estimated that the amount of information stored in living systems is 24-25 orders of magnitude larger than that of open physical systems observed in the environment. Such a huge difference stems from the fact that memory in living cells (DNA and proteins) are microscopic, while memory and self-organization units of abiotic processes are all macroscopic. A direct consequence of this difference is that regulatory capacity of living system is much larger than that of non-living systems. According to the second law of thermodynamics, to maintain a level of orderliness (amount of information) comparable to living phenomena, an abiotic system requires a prohibetively large amount of external energy.

Moreover, living systems can maintain such high level of orderliness during, in principle, unlimited periods of time. This is achieved through competitive interaction, another unique feature of life. Relatively disordered living systems (sick systems) are continually replaced by highly ordered ones (healthy systems). Although the level or orderliness of any individual organism inevitably decays following the second law of thermodynamics, the global orderliness in a population of organisms can be maintained since disordered organism are continually replaced by highly ordered ones.

From a different perspective A. Kleidon, among other authors, have highlighted two additional key properties of life regarding its significant influence on the non-equilibrium thermodynamics of the ES:

(6) Life as any other complex systems with sufficient degrees of freedom, obeys the Maximum Entropy Principle, maintain a steady state at which entropy production is maximized (Schrödinger 1992; Lorenz 2002; Ozawa et al. 2003; Kleidon and Lorenz 2005).
(7) Life (in particular photosynthetic living organisms) "generates substantial amounts of chemical free energy which essentially skips the limitations and ineffeciencies associated with the transfer of power within the system" (Kleidon 2010a).

After analysing the entropy balance of the Earth as a coupled, hierarchical and a non-equilibrium thermodynamic system it has became apparent that a widespread biota plays a driving role at generating and maintaining the habitable equilibrium state of the system (Kleidon 2010a, b, 2012). There is no reason to think that this driving role will not be also present on other inhabited planets. If so, this theoretical result agrees with the notion that the equilibrium state of an inhabited planetary environment cannot be predicted without taking into account the biotic activity.

It is worth noticing here that properties (1)-(7), although identified after studying Earth's life, are not tightly coupled to a specific model of life. Instead, they are rooted on very basic physical principles valid elsewhere in the Universe. In other words, the definition of an InHZ is supported on general properties of life as a physical complex phenomenon and not only on life as we know it on Earth.

\section{Towards a quantitative model of the InHZ}

The conceptual basis constructed over the preceeding definitions, theoretical arguments and observational evidence, is essentially aimed supporting the construction of quantitative specific models of the HZ for Inhabited Planets. Although models including biota-environment interactions have been extensively developed and applied to study the ES (see e.g. Lenton and von Bloh (2001) and references there in) the case of potentially inhabited extrasolar planets has been much less studied.

Here we present two examples of how can we asses the estimation of the InHZ limits. Limited by the huge complexity of fully-fledged models, we present here two simple albeit illustrative examples. A conceptual experiment showing how an otherwise improbable environmental state, becomes plausible under the complex dynamics of an inhabited environment. This conceptual experiment is aimed to illustrate and reinforce the argument in section 3.2. Then we present simulation results of an idealized inhabited environment, a recent variant of the "Daisyworld". There we also review the results from many other variants of this model to the light of the InHZ definition.

\subsection{The Inhabited Greenhouse-Albedo Cycler}

The dynamics of complex systems can exhibit equilibrium states (attractors) that are fixed points or limit cycles. We refer to limit cycles as states characterized by non negligible oscillations. Most of the models of the AHZ (e.g. Kasting et al. 1993 or Selsis et al. 2007) rely on fixed point 
planetary equilibrium states. This is the case, for instance, of habitable planets in the innermost edge of the RHZ in Selsis et al. (2007). Those planets remain habitable due to a constant $100 \%$ covering of water clouds.

But, what would happen if the habitable state of an inhabited planet is a limit cycle instead of a fixed point? The complex interaction between life and its abiotic environment very oftenly gives rise to natural oscillations in the environment. As a matter of fact limit cycles are the rule and not the excepton in life-bearing dynamical systems (see e.g. Nicolis and Portnow 1973). One good example of this is the seasonal cycle of $\mathrm{CO}_{2}$ in Earth's atmosphere (see e.g. the "Keeling curve" Keeling 2008) which is driven by seasonal changes in Earth's vegetation (see, e.g. Keeling et al. 1996).

Could an inhabited planet under plausible oscillating conditions be able to stretch out the AHZ limits?. In the following paragraphs we provide an example of such a plausible enhanced inhabited habitability equilibrium state.

Let us assume a hypothetical planet that remains mostly covered by water clouds only in the sunlit hemisphere while permanently uncovered by them in the opposite dark hemisphere (see Figure 2). If the planet is rotating at a faster rate than the orbital angular velocity (i.e. a non-tidally-locked planet), it will reach an oscillating equilibrium state, i.e. a limit cycle (lowest diagram in Figure 2).

In a dynamical state, clouds are continuously formed and destroyed in the atmosphere. This implies vertical transport of water. Therefore, the presence of clouds in a given place in the atmosphere implies also the presence of water along the entire atmospheric column. In such a state, less (more) clouds will necessarily imply a weaker (stronger) greenhouse effect. In our hypothetical planet the absence of clouds in the dark hemisphere makes it plausible to assume that the IR opacity of the atmosphere on that side will be less than it would be if fully covered by clouds. Greenhouse effect (GHE) will then be reduced and heat will more easily escape from the dark than from the sunlit hemisphere. On the other hand, the permanently cloud covered sunlit hemisphere, although more opaque to IR, will also have an increased albedo. If we assume that the cloud-forming effects of life (evapotranspiration, ET, and the release of organic cloud condensation nuclei, $\mathrm{CCN}$ ) only affect the formation of low altitude clouds, a sky permanently covered with this type of clouds will produce a net cooling effect on the sunlit hemisphere.

At a given point in the planetary surface, the cloud covering and water content and consequently the albedo and greenhouse effect, will oscillate as the planet rotates (we assume the planet is not a tidally-locked planet). The net effect will be to maintain every point as cool as possible combining the most favorable effect at the right hour of the day: higher albedo at noon, and reduced greenhouse effect at midnight. As a results our planet would potentially reach in this oscillating state, average surface temperatures lower than a planet fully covered by clouds.

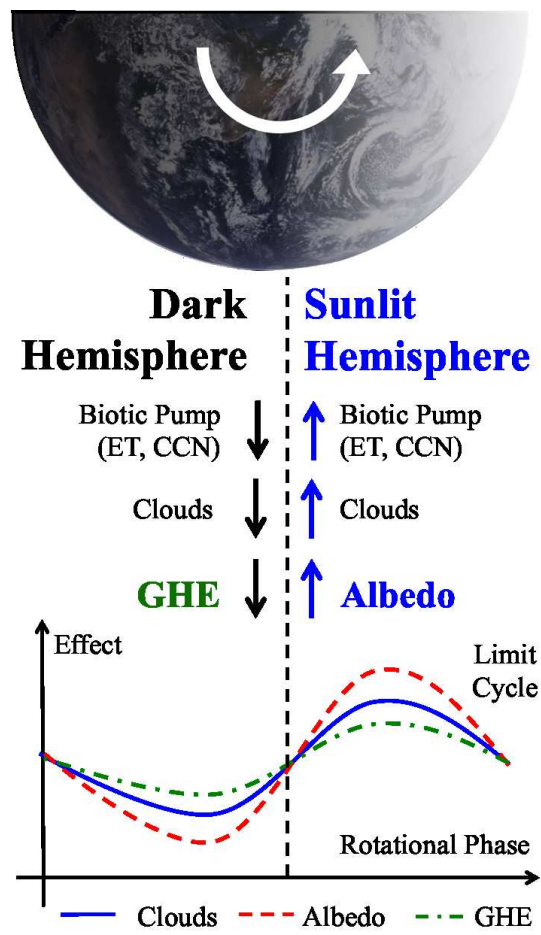

Fig. 2. The "Inhabited Greenhouse-Albedo Cycler", a hypothetical inhabited planet maintained by the complex interaction between life (referred here as the biotic pump) and its environment in a limit cycle where clouds mostly cover only the sunlit hemisphere. As the planet rotates clouds and water vapor moves through the planetary surface creating enhanced habitability conditions. While the sunlit hemisphere is cooled by the higher albedo of clouds the dark hemisphere radiates more easily the accumulated heat to space due to a reduction in the Greenhouse effect (GHE). ET refers to EvapoTranspiration and CCN to Cloud Condensation Nuclei, two of the by-products of biotic activity that strongly affects the formation of water clouds.

In terms of $\mathrm{HZ}$ limits, at distances where even $100 \%$ cloud covered planets are uninhabitable, fast rotating inhabited planets with a half cloud covered hemispheres controlled by the complex interaction between life and the environment, could be cool enough to support life. In other words the $\mathrm{HZ}$ of this "Inhabited Greenhouse-Albedo Cyclers" could be strecthed out towards the star. Figure 2 summarize schematically the dynamics of this hypothetical inhabitabed habitable planet.

In summary, under the same external forcing where abiotic (fixed point) equilibrium states are uninhabitable, an inhabited planet could achieve a habitable state through a plausible limit cycle resulting from the interaction between abiotic and biotic processes.

\subsection{A toy model of the InHZ}

One of the best known and extensively used toy model of an inhabited planet is the Daysiworld (DW) model. Originally 
introduced by Watson and Lovelock (1983), the DW model is intended to simulate the dynamics of a hypothetical simplified inhabited environment. In the model, instead of numerous variables describing the state of what otherwise would be a very complex system, only one state variable is considered: surface temperature. Moreover the biota is greatly simplified to contain only two types of living organisms: black and white daysies (Lenton and Lovelock 2001).

DW model was originally conceived as a parable with possible implications for Earth science. Its spirit is that of answering "what if...?" questions regarding the interactions between life and the environment (Lenton and Lovelock 2001). We will use here DW models in the same spirit. Thus, for instance, since the results of our DW model shows that the interaction between biota and its environment is able to modify the limits of the AHZ what would be life able to do, in more complex models. We will come back on this question later on.

Many variants of the original DW have been developed. Each of them have been intended to study different aspects of the role of life at regulating the environment (Lenton and Lovelock 2001; Wood et al. 2008). Although well-known and widely described in literature, we will briefly present here the general features of the DW model, focusing especially on the recent variant developed by Salazar and Poveda (2009). In their variant Salazar and Poveda (2009) introduced the interaction between life and the hydrological cycle. As we will show here, this key interaction is the determinant driver of planetary habitability.

The DW lies on the surface of a hypothetical Earth-sized planet, orbiting a star that provides all the required energy for an inhabited environment. The surface of the planet is partially covered by two different species of "daisies" differing, among other properties, in albedo. The original DW and many of its variants considers only "black" and "white" daisies (dark and light vegetated types of land cover). In other variants additional species having intermediate albedos are also introduced. At any time the surface of the planet is covered by variable fractions of daisies and/or bare ground.

The most important environmental variable in the model, the surface temperature $T_{s}$, is described by the global energy balance and the population-dynamics of the simplified biota (daisies). In the variant of Salazar and Poveda (2009) variant, the role of clouds and that of a global hydrologic cycle are also included in this balance. On a planet covered by fractions $a_{c}, a_{w}, a_{b}$ and $a_{d}$ of clouds, white and black daisies and bare-ground, respectively, the energy balance is granted if:

$c_{p} \frac{d T_{s}}{d t}=\frac{S S_{\odot}}{4}\left(1-\alpha_{c} a_{c}\right)\left(1-\alpha_{s}\right)+\sigma T_{c}^{4} a_{c}-\sigma T_{s}^{4}$.

Here $S$ is the stellar energy flux measured in units of the solar flux at Earth distance (Solar constant, $S_{\odot}$ ), $c_{p}$ is the planetary mean heat capacity or thermal inertia and $\sigma$

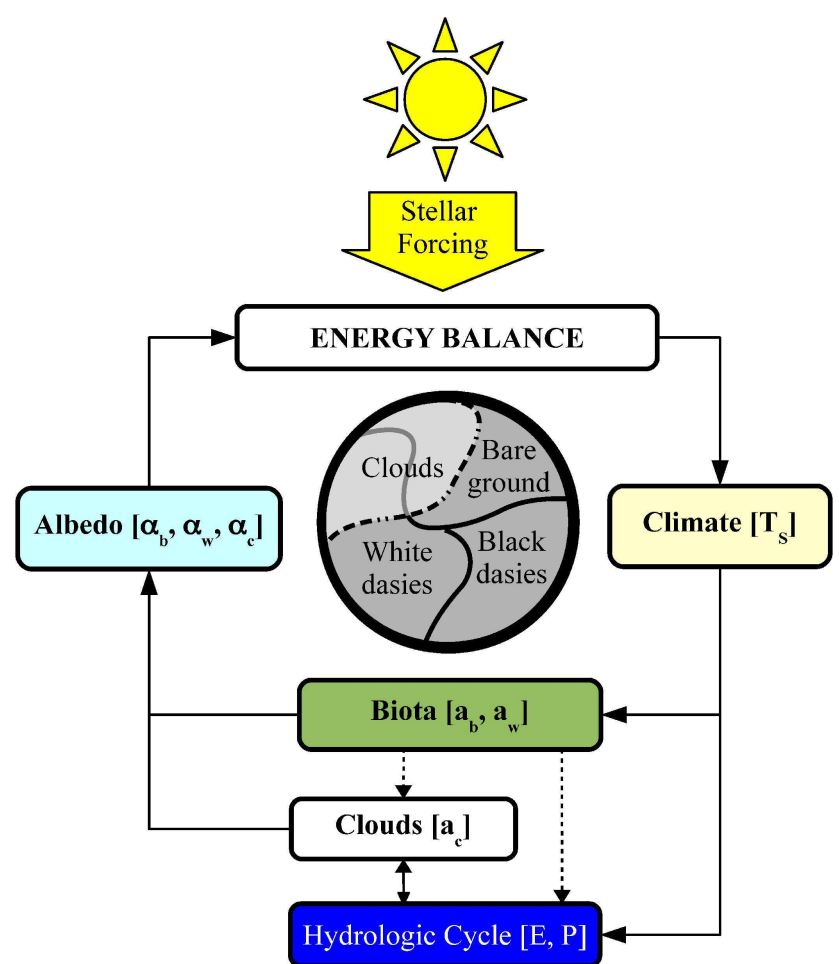

Fig. 3. Schematic representation of a DW model including clouds and a hydrologic cycle (Salazar and Poveda 2009). Components of the system (subsystems and processes) are indicated inside rectangles. Basic properties associated to each component are written in brackets (see equations in the main text). Feedbacks between components are indicated with arrows. Dashed arrows going from biota to clouds and to hydrologic cycle are hypothetical feedbacks not included yet in the DW variant studied here.

is the Stefan-Boltzmann constant. $\alpha_{s}$ and $\alpha_{c}$ are the albedo of planetary surface and clouds, respectively. $T_{c}$ is the temperature of clouds which is related to $T_{s}$ through the atmospheric lapse rate (Salazar and Poveda 2009). Total surface albedo depends on the area fractions of black daisies, white daisies and bare ground, whose albedos are denoted by $\alpha_{b}$, $\alpha_{w}$, and $\alpha_{d}$ respectively. We are adopting here for the stellar adimensional irradiance $S$, the notation commonly used in habitability literature instead of that used in DW literature where $L$ is used to denote this quantity. It should be noticed that removing clouds $\left(a_{c}=0\right)$ and giving the planet a null thermal inertia $\left(c_{p}=0\right)$ leads to an exact radiative balance between the net incident short-wave and outgoing longwave radiation, i.e. $\left(S S_{\odot} / 4\right)\left(1-\alpha_{s}\right)=\sigma T_{s}^{4}$ which correspond to the energy balance equation in the original DW model (Watson and Lovelock 1983) and most of its variants.

The evolution of daisies populations is given by the growth equations (Carter and Prince 1981),

$\frac{d a_{i}}{d t}=a_{i}\left[\left(1-a_{w}-a_{b}\right) \beta_{i}-\gamma\right]$, 
where the subscripts, $i=b, w$ refer to black $(b)$ and white $(w)$ daisies. Here $\beta_{i}$ is the growth rate of the $i$-daisies which is a function of the planetary temperature and other parameters conceptually related to their biology (e.g. tolerance to high temperatures, adaptation, symbiosis); $\gamma$ is the mortality rate commonly assumed as constant for both type of daisies. Different variants of DW use modified version of Eq. (2) and different functions $\beta_{i}$ intended to simulate certain aspects of biological dynamics such as biodiversity (Lovelock 1992), adaptation (Lenton and Lovelock 2000; Roberston and Robinson 1998), different forms of competition (Cohen and Rich 2000), or symbiosis (Boyle et al. 2011).

The hydrologic cycle, included for completeness in our description of the model and first introduced by Salazar and Poveda (2009), is described with the mass balance equation:

$\frac{d a_{c}}{d t}=E-P$,

where $a_{c}$ is assumed proportional to the atmospheric water content and $E=\left(1-a_{c}\right) E_{p}$ and $P=a_{c} P_{p}$ are the actual evapotranspiration and precipitation rates. For a detailed description of these factors please refer to Salazar and Poveda (2009).

Figure 3 depicts schematically the feedbacks at play in a DW model including clouds and a hydrologic cycle. The only external forcing in the model is the stellar insolation. In the inner loop (energy balance - climate - biota - albedo) the energy balance between incoming and outgoing radiation determines surface planetary temperature which in turn influences daisies population (biota) changing planetary albedo. Albedo finally enters into the energy balance closing the loop. When including the effect of the hydrologic cycle and clouds, surface temperature also affects the exchange of water between the surface and the atmosphere through precipitation and evapotranspiration. The presence of clouds in the atmosphere depends on such processes and viceversa. Clouds influences also planetary albedo (and greenhouse effect) which in turn determine the energy balance at the top, closing the loop. Since habitability depends directly on surface temperature, habitable equilibrium states in DW models will depend on the complex relationship among all these feedbacks. For completeness, two hypothetical feedbacks (dashed arrows in Figure 3), not yet included in any DW variant, have been also depicted in our schematic representation. These feedback arises from the biota-cloud and biota-hydrologic cycle interactions, and they have been mentioned in detail in Section 3.1 in the case of the ES. They are recognized as potential key drivers of regulatory dynamics in a inhabited planet and should be included in future improved biota-environment models.

To illustrate the emerging properties of a DW with a hydrological cycle we show in Figure 4 a typical result of

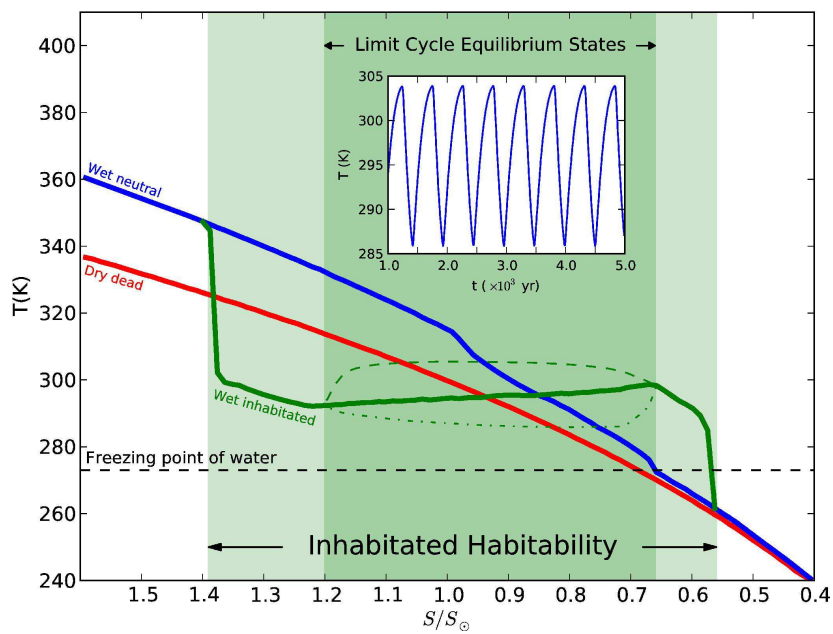

Fig. 4. Equilibrium temperature versus stellar radiative forcing in a DW model including clouds and a hydrological cycle (green solid curve). For comparison the equilibrium temperature of the planet when uninhabited (red and blue lines are also included). In this particular case the presence of life places the outer limit of InHZ further away from the star. Compare the place where green, blue and red lines crosses the freezing temperature of water (black dashed line) at low stellar insolations.

solving the DW equations for different stellar insolations (Salazar and Poveda 2009). We plot there the equilibrium temperature as a function of the input stellar irradiance for the model also depicted in Figure 7 of Salazar and Poveda 2009. In this case we have assumed a DW covered by clouds with a 0.6 albedo located at $4 \mathrm{~km}$ above planetary surface. This clouds resembles the mid altitude clouds in the Earth. We have verified that the conclussions drawn from this example will not change too much when changing other model parameters.

In the figure the difference between the equilibrium surface temperature when dead, i.e. uninhabited (curves marked as wet neutral and wet dry) and when inhabited, is notorious, especially in the solar forcing range $S / S_{\odot}=0.56-1.39$. This is precisely what we can call here the Inhabited Habitable Zone of the system. In this range of solar forcing, surface temperatures stay regulated around the temperatures where the growth of daisies is optimum $\left(T_{s}=20-25 \mathrm{C}\right)$.

Without life a stellar forcing of for example $S / S_{\odot}=0.6$ will produce an equilibrium state (with and without clouds and greenhouse effect) characterized by surface temperatures below the freezing point of water (uninhabitable planet). With the same stellar forcing the biota-environment system reaches an equilibrium state with temperatures almost 20 degrees above the freezing point of water.

More interestingly is the interval of $S / S_{\odot}=0.66-1.20$ the equilibrium state of the system in that range is not a fixed point but a limit cycle characterized by temperature oscillations with a significant constant amplitude and mean value 
(inset panel). Temperatures in these oscillatory states are represented in the figure by their mean (wet inhabited solid line), maximum (dashed line) and minimum (dash-dotted line) values. The inset figure shows the oscillations corresponding to $S / S_{\odot}=1$. Since the occurence of such oscillations depends on the biota-environment interactions, the range of values of $S$ where oscillations arise, is inside the InHZ. It is interesting to notice however that not all the equilibrium states inside the InHZ are limit cycles. For example at minimum and maximum values of $S$ the equilibrium states are actually fixed points. This behavior arises from the fact that at those extremes the interspecific competition dissapears. This is due to the fact that only one the species is inhabitating the planet. Table 2 in Salazar and Poveda (2009) shows that for a wide range of model parameters the equilibrium states are limit cycles. This confirms the idea that such type of oscillating behavior in system with complex biota-environment interactions are the rule rather than the exception. The noticeable prevalence of limit cycles in DW is in agreement with the previous results by Nevison et al. (1999) and support the hypothesis behind the conceptual experiment discussed in Section 4.1 .

To summarize the evidence coming from a wide diversity of DW models supporting the InHZ concept, we present in Table 1 the limits of the $\mathrm{HZ}$ as calculated using different assumptions about biota properties and its interspecific interactions. This table is a modified extension of that published by Lenton and Lovelock (2001). A graphical representation of these results are presented in Figure 5. We have used there the common graphical representation of the circumstellar habitable zone representing curves of constant $S$ in a plane of $T_{\text {eff }}$ vs. $a$ (stellar effective temperature vs. planet-star distance). It is important to stress that although using the same graphical representation, a direct comparison among Figure 5 and a similar one representing the limits of the AHZ as calculated for example with 1-D atmospheric models (e.g. Kopparapu et al. (2013)) is not straightforward. It is worth to recall here DW models use very simplified (if not unexistent) models for the atmopsheric response to the incoming stellar radiation and depend on even simpler models of what would be very complex biota-environment interactions. The Inhabited Habitable Zones in Figure 5 are a parable of real InHZs in the same way as DW is a parable of the ES.

The results compiled in Table 1 and represented in Figure 5 clearly show that the limits of the HZ are sensitive first to the presence of life (the width of the HZ in planets with biotaenvironment interactions are at the least two times wider than that corresponding to a neutral planet), and second to the particular properties of life and its interaction with the environment. Thus, for example giving some adaptation capabilities to daisies (see Constrained Adaptation InHZ) widens the $\mathrm{HZ}$ span with respect to other DW models where the intrinsic properties of life are constant and independent of the environment (see e.g. Original InHZ). These results lend support

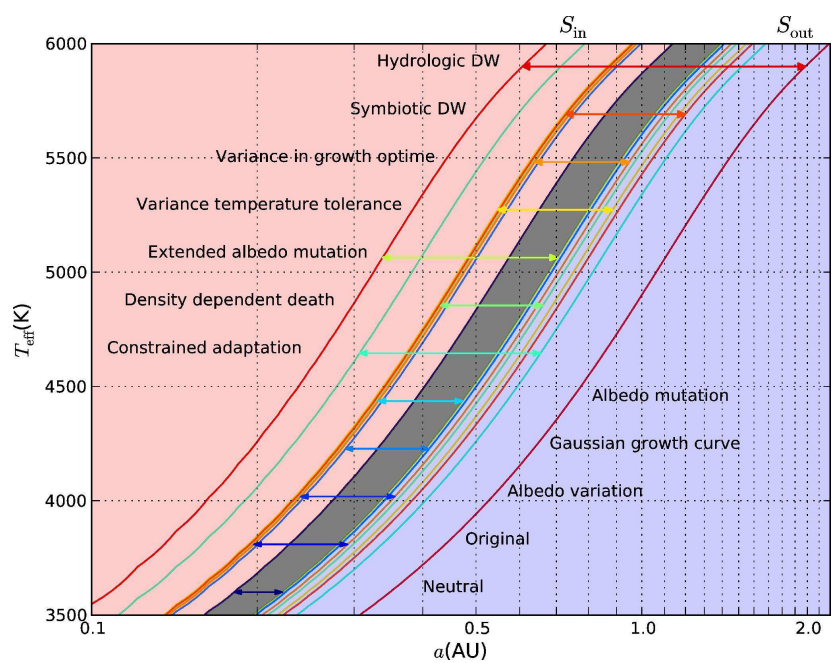

Fig. 5. Inhabited Habitable Zones for different variants of the DW model (see Table 1). Curves indicate the distance $a$ to a main sequence star with effective temperature $T_{\text {eff }}$ where equal incoming flux $S / S_{\odot}$ is received in the inner and outer InHZ edges. Labels summarize the criteria distinguishing each variant. The length of the double arrow in the middle of each strip represent the span $S_{\mathrm{R}}$ of the InHZ.

to the idea that the InHZ could be significantly different (and probably much wider) than the AHZ.

\section{Discussion}

The theoretical arguments, conceptual experiments and numerical models presented here have esatblished a minimum conceptual framework on which justifying and building planetary habitability models including the unavoidable effect of life itself. However, an in depth approach to the estimation of a realistic InHZ, will require the assesment of several key issues not discussed yet. Although an exhaustive enumeration and discussion of the the many aspects involved in this problem is certainly out of the scope of this paper, we will try to summarize here some of the most important open issues that should be addressed in forecoming papers.

At which extent the limits of the InHZ will depend on the very specific properties of life inhabiting a given planetary environment? In other words, are the InHZ limits different for each type of organisms? Will each form of life define a different InHZ even within the same planetary system?

As discussed in Section 2 the very definition of the InHZ depends on general properties of life as a complex physical phenomenon despite its specific traits. However, it is also clear that different "models of life" could be characterized by different optimum physical conditions where it could thrive (see for instance the differences between the InHZ limits of the DW models in Figure 5). It would be thus obvious that each model of life will determine different InHZ limits. How- 
Table 1. Inner limit $S_{\text {in }}$, outer $S_{\text {out }}$ limit and span $S_{\mathrm{R}}=S_{\text {out }}-S_{\text {in }}$ of the InHZ for different variants of the DW model. Adapted from Lenton and Lovelock (2001).

\begin{tabular}{llccc}
\hline Reference & Criterion & $S_{\text {out }}$ & $S_{\text {in }}$ & $S_{R}$ \\
\hline \hline Watson and Lovelock 1983 & Neutral Daisy & 0,74 & 1,11 & 0,37 \\
& Original Daisyworld & 0,68 & 1,50 & 0,82 \\
\hline Lovelock 1989 & Albedo variation & 0,68 & 1,50 & 0,82 \\
\hline Harting and Lovelock 1996 & Gaussian growth curve & 0,72 & 1,45 & 0,73 \\
\hline Lenton 1998 & Albedo mutation & 0,74 & 1,50 & 0,76 \\
\hline Lenton and Lovelock 2000 & Constrained adaptation & 0,51 & 2,32 & 1,81 \\
\hline Lenton and Lovelock 2001 & Density dependent death & 0,65 & 1,53 & 0,88 \\
& Extended albedo mutation & 0,74 & 3,20 & 2,46 \\
& Variance in Temperature tolerance & 0,60 & 1,57 & 0,97 \\
& Variance in growth optima & 0,68 & 1,50 & 0,82 \\
\hline Boyle et al. 2011 & Symbiotic DW increasing luminosity & 0,57 & 1,55 & 0,98 \\
\hline Salazar and Poveda 2009 & Hydrologic Daisyworld & 0,30 & 3,26 & 2,96 \\
\hline \hline
\end{tabular}

ever, an analogous situation arises in the definition of the RHZ when considering for instance different types of clouds or different geological and geodynamical conditions. In analogy to what is done at defining the more general RHZ, we should choose the most general or common traits we could expect for most forms of life thriving in the Universe.

But, how many different forms of life could exist out there? Are they knowable even in principle? Although we are far from solving these questions, we can still make some efforts for calculating the InHZ limits of the Solar System and elsewhere, at least for the type of life we know on Earth.

Even if we calculate an "Earth-life-like" InHZ in an extrasolar planetary system, it would be possible that other models of life (for instance "extremophiles biotas") able to contribute at establishing habitable equilibrium states beyond those limits, still exist. This case, however, will not be too different from the case when we can find extremophile organisms that are able to thrive beyond the limits of the AHZ. However, as opposed to extremophile organisms, extreme biotas will thrive in planets actually habitable for a large range of organisms (extremophiles or not).

One of the most interesting astronomical consequences of introducing the concept of an InHZ is that habitability instead of being a prerrequisite for life could be actually a proxy for an inhabited environment. In other words, the detection of a habitable planet could be the confirmation that life actually exist on its surface. The key property of habitable environments enabling this possibility is its intrinsic instability. Let us illustrate this with an important example: the existence of a hydrosphere.

Since the seminal works on planetary habitability by Shapley (1953) and Hart (1979), the presence of a liquid hydrosphere has been regarded as a prerequisite for life as we know it. However it has been shown that a planetary equilibrium state including a liquid hydrosphere is highly unstable (Gorshkov and Makarieva 2002; Gorshkov et al. 2004).
In such a state and in the absence of powerful regulating feedbacks, the system will rapidly make a transition to equilibrium stable states where the hydrosphere evaporates causing a catastrophic greenhouse effect or it completely freezes out. Obviosuly both states are prohibitive for life. The origin of such instability is the positive feedback affecting the equilibrium amount of atmospheric water vapour and its greenhouse effect. In the presence of a hydrosphere, evaporation will increase the atmopsheric water vapour thus increasing the greenhouse effect and surface temperatures that at its turn increases even more the evaporation rate and so on. On the other hand frozen water have a larger albedo than liquid water which tends to reduce average temperatures increasing the frozen water in the planet and so on.

Gorshkov et al. (2004) calculated that if left unregulated, the Earth's hydrosphere will be fully evaporated or frozen in less than $10^{4}$ years. Therefore, in order to maintain a global hydrosphere during geological timescales, the Earth could have required powerful regulatory mechanisms. We have already argued how life overcomes by many orders of magnitude the reglatory power of other abiotic processes. In this sense, and as stated before, the presence of a liquid hydrosphere instead of being a prerrequisite for life can be actually regarded as a proxy for the presence of life itself. In practical terms, detecting oceans and other large masses of liquid water in the surface of extrasolar planets, instead of pointing to the possibility that life could thrive on the planet, would be actually a signature of the actual presence of life. Oceans could be the ultimate biosignature.

Is the InHZ concept tightly bound to Gaia or BR theories? Not at all. Although both theories provide very important arguments and evidences supporting the definition of an InHZ, stating that life is an important factor that could not be disregarded at calculating the equilibrium state of a habitable planet is very different than assuming that life is the most important one. Our point here is that in a complex system such 
as an inhabited planet taking away any major component of the system gives you back another planet. In simple words, the whole without its $10 \%$ is not $90 \%$ of the whole (here $10 \%-90 \%$ could be replaced by $50 \%-50 \%$ or $90 \%-10 \%$ )

Are the DW model the only way to approach to the quantitative determination of the InHZ limits? Definitevly no. Actually the best way to approach to this complex problem is by modelling in full detail the complex interactions and feedbacks between life and its abiotic environment on our own Earth as a first example. Very complex models of this sort have been developed in the past. Our task now will be to apply those models not for studying the Earth but for looking for other Earths in the Universe.

\section{Summary and Conclusions}

We have presented here theoretical arguments supporting the idea that life cannot be excluded when finding the plausible equilibrium states that define the limits of the Habitable Zone. Since our final goal at searching for habitable planets is precisely looking for the inhabited ones, turning from the traditional definition of an AHZ to a more general InHZ is mandatory. The arguments presented here were based on mounting observational evidence as well as on theories developed along the last decades, supporting the idea that life have a non-negligible effect on the environment of the only habitable planet we know so far: the Earth. Although including life in all its complexity in a realistic model of the environment of any inhabited planet is challenging, we have shown trhough simple albeit illustrative conceptual and numerical experiments that it can be achieved. More importantly, we showed that life is able to substantially modify the limits of the otherwise uninhabited AHZ and therefore, potentially expand the region in the parameter space where we are presently searching for it. Together, the theoretical arguments, the observational evidences, and the simple examples provided here, constitute a general conceptual framework on which more complex models of the InHZ can be developed.

The InHZ, as defined here, is the region where the interaction between life and its abiotic planetary environment supports the necessary physical conditions for the very existence and persistence of life itself. This concept is in starking contrast with the definition of an AHZ which is commonly used in astrobiology and exoplanetary research. Our work emphasizes the fact that habitability is an emergent property of the complex biota-environment coupled system and not simply a physical prerrequisite entirely determined by astrophysical, geophysical and other abiotic factors.

We did not address here the problem of the origin of life at defining the InHZ in the same way as the ideas of an abiotic habitability have never addressed the problem of the origin of liquid water. In other words, we argue that answering the question "which comes first, life or habitability?" is analogous to trying to answer the question "which comes first, liquid water or habitable surface temperatures?". Complex systems such as habitable planets or in general planetary environments are characterized by this sort of what should be considered fake egg-and-chicken paradoxes. The origin of emergent properties in complex system does not require simple sequential explanations.

Our definition of an InHZ should not be confused with a definition based on the capacity of extremophiles organisms to thrive under conditions beyond the limits of the living organisms on Earth. By definition, an inhabited habitable planet should guarantee habitable conditions to all organisms able to thrive in the range of environmental conditions characterizing the equilibrium state of the planet. If these equilibrium conditions are extreme for Earth organisms it does not make life in this planet extremophilic but just different. In the same line of reasoning, our InHZ definition does not consider the case of hidden biospheres (e.g. life thriving in the solid or liquid planetary interior). As usual at defining the InHZ we are looking for life able to produce detectable signatures in the planetary atmosphere and/or its surface.

Our aim here was not to provide limits of the InHZ but to pose the question what if habitable zone models are excluding a key component of the planetary environment. In that sense our central point should be read not as a prove but as a question. Paraphrasing the Einstein's quote at the beginning of this paper "we should make things as simple as possible, but not simpler". When dealing with habitability we should consider as few factors as possible but not fewer. Life is certainly an unavoidable factor.

The InHZ concept could be further explored in several directions and may serve as a conceptual framework for developing more realistic models of planetary habitability. For instance, we can try to introduce already known biotic feedbacks in some widely accepted models of abiotic habitability. On the other hand we can improve the most simple DW models including a more realistic treatment of the response of the atmosphere to solar forcing. Although the models by Salazar and Poveda (2009) were aimed in that direction, further efforts to improve their atmospheric model should be pursued. Evolution is a key process for life and its role at determining the way as biota alter its environment would also be a key step towards more realistic InHZ models.

Acknowledgements. We want to especially thank to our colleagues Victor Gorshkov, Anastassia Makarieva, Rene Heller, Dave Waltham and Peter Bunyard for a preliminary revision of the manuscript. Their insightful comments, corrections and clever questions were fundamental at determining the final version of the manuscript. JZ thanks the AMEBA group for inspiring some of the key ideas in this work and for allowing us to discuss some of them in its weekly meetings. This work has been done with the financial support of CODI-UdeA. 


\section{Auhtor disclosure statement}

All the authors manifest that no competing financial interests exist in connection with the ideas published in this manuscript.

\section{References}

Andreae, M., Rosenfeld, D., Artaxo, P., Costa, A., Frank, G., Longo, K., and Silva-Dias, M.: Smoking rain clouds over the Amazon, Science, 303, 1337-1342, 2004.

Arneth, A., Harrison, S., Zaehle, S., Tsigaridis, K., Menon, S., Bartlein, P., Feichter, J., Korhola, A., Kulmala, M., O'donnell, D., et al.: Terrestrial biogeochemical feedbacks in the climate system, Nature Geoscience, 3, 525-532, 2010.

Batalha, N. M., Rowe, J. F., Bryson, S. T., Barclay, T., Burke, C. J., Caldwell, D. A., Christiansen, J. L., Mullally, F., Thompson, S. E., Brown, T. M., et al.: Planetary Candidates Observed by Kepler. III. Analysis of the First 16 Months of Data, The Astrophysical Journal Supplement Series, 204, 24, 2013.

Beerling, D.: Leaf evolution: gases, genes and geochemistry, Annals of Botany, 96, 345-352, 2005.

Bonan, G.: Forests and climate change: forcings, feedbacks, and the climate benefits of forests, science, 320, 1444-1449, 2008.

Boyle, R., Lenton, T., and Watson, A.: Symbiotic physiologypromoteshomeostasisinDaisyworld, Journal ofTheoreticalBiology, 274, 170-182, 2011.

Caldeira, K., Kasting, J., et al.: The life span of the biosphere revisited, Nature, 360, 721-723, 1992.

Carter, R. N. and Prince, S. D.: Epidemic models used to explain biogeographical distribution limits, Nature , 293, 644-645, 1981.

Cohen, J. and Rich, A.: Interspecific competition affects temperature stability in Daisyworld, Tellus B, 52, 980-984, 2000.

DeLeon-Rodriguez, N., Lathem, T. L., Rodriguez-R, L. M., Barazesh, J. M., Anderson, B. E., Beyersdorf, A. J., Ziemba, L. D., Bergin, M., Nenes, A., and Konstantinidis, K. T.: Microbiome of the upper troposphere: Species composition and prevalence, effects of tropical storms, and atmospheric implications, Proceedings of the National Academy of Sciences, 110, 25752580, 2013.

Dole, S. H.: Habitable planets for man, Cambridge, 1964.

Dyke, J., Gans, F., and Kleidon, A.: Towards understanding how surface life can affect interior geological processes: a nonequilibrium thermodynamics approach, Earth System Dynamics, 2, 139-160, 2011.

Foley, J. A., Costa, M. H., Delire, C., Ramankutty, N., and Snyder, P.: Green surprise? How terrestrial ecosystems could affect earth's climate, Frontiers in Ecology and the Environment, 1, 3844, 2003.

Franck, S.: Global water cycle and Earth's thermal evolution, Journal of Geodynamics, 32, 231-246, 2001.

Franck, S., Kossacki, K., and Bounama, C.: Modelling the global carbon cycle for the past and future evolution of the earth system, Chemical Geology, 159, 305-317, 1999.

Franck, S., Block, A., von Bloh, W., Bounama, C., Schellnhuber, H.-J., and Svirezhev, Y.: Habitable zone for Earth-like planets in the solar system, Planet. Space Sci. , 48, 1099-1105, 2000a.
Franck, S., von Bloh, W., Bounama, C., Steffen, M., Schönberner, D., and Schellnhuber, H.-J.: Determination of habitable zones in extrasolar planetary systems: Where are Gaia's sisters?, J. Geophys. Res. , 105, 1651-1658, 2000 b.

Franck, S., Block, A., Bloh, W., Bounama, C., Garrido, I., and Schellnhuber, H.-J.: Planetary habitability: is Earth commonplace in the Milky Way?, Naturwissenschaften, 88, 416-426, 2001.

Gorshkov, V.: Physical and biological bases of life stability. Man, biota, environment, Springer, 1995.

Gorshkov, V. and Makarieva, A.: On the possibility of physical selforganization of biological and ecological systems, Doklady Biological Sciences, 378, 258-261, 2001.

Gorshkov, V. and Makarieva, A.: Greenhouse effect dependence on atmospheric concentrations of greenhouse substances and the nature of climate stability on Earth, Atmospheric Chemistry and Physics Discussions, 2, 289-337, 2002.

Gorshkov, V. G., Gorshkov, V. V., and Makarieka, A. M.: Biotic regulation of the environment, Springer, 2000.

Gorshkov, V. G., Makarieva, A. M., and Gorshkov, V. V.: Revising the fundamentals of ecological knowledge: The biotaenvironment interaction, Ecological Complexity, 1, 17-36, 2004.

Hart, M. H.: Habitable Zones about Main Sequence Stars, Icarus , 37, 351-357, 1979.

Harting, S. and Lovelock, J.: Exploiter-mediated coexistence and frequency-dependent selection in a numerical model of biodiversity, J. Theoretical Biology, 182, 109-116, 1996.

Hitchcock, D. R. and Lovelock, J. E.: Life detection by atmospheric analysis, Icarus , 7, 149-159, 1967.

Höning, D., Hansen-Goos, H., Airo, A., and Spohn, T.: Biotic vs. abiotic Earth: A model for mantle hydration and continental coverage, Planetary and Space Science, 2013.

Houghton, J., Ding, Y., Griggs, D., Noguer, M., van der LINDEN, P., Dai, X., Maskell, K., and Johnson, C.: Climate change 2001: the scientific basis, Cambridge University Press Cambridge, 2001.

Hutjes, R., Kabat, P., Running, S., Shuttleworth, W., Field, C., Bass, B., da Silva Dias, M., Avissar, R., Becker, A., Claussen, M., et al.: Biospheric aspects of the hydrological cycle, Journal of Hydrology, 212, 1-21, 1998.

Jasechko, S., Sharp, Z. D., Gibson, J. J., Birks, S. J., Yi, Y., and Fawcett, P. J.: Terrestrial water fluxes dominated by transpiration, Nature, 2013.

Kasting, J.: How to Find a Habitable Planet, Princeton University Press, 2010.

Kasting, J. F., Whitmire, D. P., and Reynolds, R. T.: Habitable Zones around Main Sequence Stars, Icarus , 101, 108-128, 1993.

Keeling, C., Chin, J., and Whorf, T.: Increased activity of northern vegetation inferred from atmospheric $\mathrm{CO} 2$ measurements, Nature, 382, 146-149, 1996.

Keeling, R.: Atmospheric science. Recording Earth's vital signs., Science, 319, 1771, 2008.

Kitzmann, D., Patzer, A., von Paris, P., Godolt, M., Stracke, B., Gebauer, S., Grenfell, J., and Rauer, H.: Clouds in the atmospheres of extrasolar planets. I. Climatic effects of multilayered clouds for Earth-like planets and implications for habitable zones, arXiv preprint arXiv:1002.2927, 2010.

Kleidon, A.: Maximum entropy production and general trends in biospheric evolution, Paleontological Journal, 43, 980-985, 
2009.

Kleidon, A.: Life, hierarchy, and the thermodynamic machinery of planet Earth, Physics of life reviews, 7, 424-460, 2010a.

Kleidon, A.: Life as the major driver of planetary geochemical disequilibrium. Reply to comments on Life, hierarchy, and the thermodynamic machinery of planet Earth, Physics of life reviews, 7, 473-476, 2010b.

Kleidon, A.: How does the Earth system generate and maintain thermodynamic disequilibrium and what does it imply for the future of the planet?, Philosophical Transactions of the Royal Society A: Mathematical, Physical and Engineering Sciences, 370, 1012-1040, 2012.

Kleidon, A. and Lorenz, R. D.: Non-equilibrium thermodynamics and the production of entropy: life, earth, and beyond, Springer, 2005.

Kopparapu, R. K., Ramirez, R., Kasting, J. F., Eymet, V., Robinson, T. D., Mahadevan, S., Terrien, R. C., Domagal-Goldman, S., Meadows, V., and Deshpande, R.: Habitable Zones around Main-sequence Stars: New Estimates, ApJ , 765, 131, 2013.

Lenton, T.: Gaia and natural selection, Nature, 934, 439-447, 1998.

Lenton, T.: Testing Gaia: the effect of life on Earth's habitability and regulation, Climatic Change, 52, 409-422, 2002.

Lenton, T.: Clarifying Gaia: regulation with or without natural selection, Scientists Debate Gaia, pp. 15-25, 2004.

Lenton, T. and Lovelock, J.: Daisyworld is Darwinian:Constraints on Adaptation are Important for Planetary Self-Regulation, J. Theoretical Biology, 206, 109-114, 2000.

Lenton, T. and Lovelock, J.: Daisyworld revisited: Quantifying biological effects on planetary self-regulation, Tellus, 53B, 288305, 2001.

Lenton, T. and von Bloh, W.: Biotic feedback extends the life span of the biosphere, Geophys. Res. Lett, 28, 1715-1718, 2001.

Lenton, T. and Wilkinson, D.: Developing the Gaia Theory. A Response to the Criticisms of Kirchner and Volk, Climatic Change, 58, 1-12, 2003.

Lorenz, R. D.: Planets, life and the production of entropy, International Journal of Astrobiology, 1, 3-13, 2002.

Lovelock, J.: A numerical model for biodiversity, Philosophical Transactions of the Royal Society of London. Series B: Biological Sciences, 338, 383-391, 1992.

Lovelock, J.: The ages of Gaia: a biography of our living earth, Oxford University Press, 1995.

Lovelock, J. E.: Gaia: A New Look at Life on Earth, Oxford University Press, 1979.

Lovelock, J. E.: Geophysiology, Transactions of the Royal Society of Edinburgh: Earth Sciences, 80, 169-175, 1989.

Lovelock, J. E.: The vanishing face of gaia: a final warning, Basic Books, New York, 2009.

Lovelock, J. E. and Margulis, L.: Homeostatic tendencies of the Earth's atmosphere, Origins of Life, 5, 93-103, 1974.

Lyons, T.: Clouds prefer native vegetation, Meteorology and Atmospheric Physics, 80, 131-140, 2002.

Makarieva, A., Gorshkov, V., Li, B., and Chown, S.: Sizeand temperature-independence of minimum life-supporting metabolic rates, Functional Ecology, 20, 83-96, 2006.

Makarieva, A., Gorshkov, V., et al.: Biotic pump of atmospheric moisture as driver of the hydrological cycle on land, Hydrology and Earth System Sciences Discussions, 11, 1013-1033, 2007.
Makarieva, A., Gorshkov, V., Sheil, D., Nobre, A., and Li, B.: Where do winds come from? A new theory on how water vapor condensation influences atmospheric pressure and dynamics, Atmospheric Chemistry and Physics Discussions, 10, 2010.

Margulis, L. and Lovelock, J. E.: Biological Modulation of the Earth's Atmosphere, Icarus , 21, 471, 1974.

Meskhidze, N. and Nenes, A.: Phytoplankton and cloudiness in the Southern Ocean, Science, 314, 1419-1423, 2006.

Mischna, M. A., Kasting, J. F., Pavlov, A., and Freedman, R.: Influence of carbon dioxide clouds on early Martian climate, Icarus, 145, 546-554, 2000.

Nevison, C., Gupta, V., and Klinger, L.: Self-sustained temperature oscillations on Daisyworld, Tellus, 51B, 806-814, 1999.

Nicolis, G. and Portnow, J.: Chemical oscillations, Chemical Reviews, 73, 365-384, 1973.

Ozawa, H., Ohmura, A., Lorenz, R. D., and Pujol, T.: The second law of thermodynamics and the global climate system: a review of the maximum entropy production principle, Reviews of Geophysics, 41, 1018, 2003.

Pöschl, U., Martin, S., Sinha, B., Chen, Q., Gunthe, S., Huffman, J., Borrmann, S., Farmer, D., Garland, R., Helas, G., et al.: Rainforest aerosols as biogenic nuclei of clouds and precipitation in the Amazon, Science, 329, 1513-1516, 2010.

Poveda, G., Jaramillo, L., and Vallejo, L. F.: Seasonal precipitation patterns along pathways of South American low-level jets and aerial rivers, Water Resources Research, 2014.

Ramanathan, V., Cess, R., Harrison, E., Minnis, P., Barkstrom, B., Ahmad, E., Hartmann, D., et al.: Cloud-radiative forcing and climate: Results from the Earth Radiation Budget Experiment., Science, 243, 57, 1989.

Rial, J., Pielke, R., Beniston, M., Claussen, M., Canadell, J., Cox, P., Held, H., De Noblet-Ducoudré, N., Prinn, R., Reynolds, J., et al.: Nonlinearities, feedbacks and critical thresholds within the Earth's climate system, Climatic Change, 65, 11-38, 2004.

Ridgwell, A. and Zeebe, R.: The role of the global carbonate cycle in the regulation and evolution of the Earth system, Earth and Planetary Science Letters, 234, 299-315, 2005.

Roberston, D. and Robinson, J.: Darwinian daisyworld, Journal of Theoretical Biology, 195, 129-134, 1998.

Rosing, M. T., Bird, D. K., Sleep, N. H., and Bjerrum, C. J.: No climate paradox under the faint early Sun, Nature , 464, 744 747, 2010.

Runyan, C., D’Odorico, P., and Lawrence, D.: Physical and biological feedbacks of deforestation, Reviews of Geophysics, 50, RG4006, 2012.

Sagan, C. and Mullen, G.: Earth and Mars: Evolution of Atmospheres and Surface Temperatures, Science, 177, 52-56, 1972.

Salazar, J. F. and Poveda, G.: Role of a simplified hydrological cycle and clouds in regulating the climate-biota system of Daisyworld, Tellus B, 61, 483-497, 2009.

Schellnhuber, H.: 'Earth system' analysis and the second Copernican revolution, Nature, 402, C19-C23, 1999.

Schimel, D.: Terrestrial ecosystems and the carbon cycle, Global change biology, 1, 77-91, 1995.

Schrödinger, E.: What is life?: With mind and matter and autobiographical sketches, Cambridge University Press, 1992.

Selsis, F., Kasting, J. F., Levrard, B., Paillet, J., Ribas, I., and Delfosse, X.: Habitable planets around the star Gliese 581?, A\&A , 476, 1373-1387, 2007. 
Shapley, H.: Climatic change: evidence, causes, and effects, Climatic Change: Evidence, Causes, and Effects, 1, 1953.

Steffen, W.: Global change and the earth system: a planet under pressure, Global change-the IGBP series (, 2004.

Walker, J. C. G., Hays, P. B., and Kasting, J. F.: A negative feedback mechanism for the long-term stabilization of the earth's surface temperature, J. Geophys. Res. , 86, 9776-9782, 1981.

Watson, A. and Lovelock, J.: Biological homeostasis of the global environment: The parable of Daisyworld, Tellus, 35B, 284-289, 1983.

Wood, A., Ackland, G., Dyke, J., Williams, H., and Lenton, T.: Daisyworld: A review, Reviews of Geophysics, 46, 1-23, 2008.

Zuluaga, J. I., Bustamante, S., Cuartas, P. A., and Hoyos, J. H.: The Influence of Thermal Evolution in the Magnetic Protection of Terrestrial Planets, ApJ , 770, 23, 2013. 